\title{
Some Restricted Partition Functions
}

\author{
Sabuj Das* \\ Senior Lecturer, Department of Mathematics Raozan University College, Bangladesh \\ *Corresponding author: sabujdas.ctg@gmail.com
}

Received April 09, 2014; Revised November 07, 2014; Accepted November 24, 2014

\begin{abstract}
In 1742, Euler found the generating function for $\mathrm{P}(\mathrm{n})$. Hardy said Ramanujan was the first, and upto now the only, Mathematician to discover any such properties of $\mathrm{P}(\mathrm{n})$. In 1952, Macmahon established a table of $\mathrm{P}(\mathrm{n})$ for the first 200 values of $n$. This paper showed how to find the number of partition of $n$ by using Macmahon's table. In 1742, Euler also stated the series in the enumeration of partitions. This Paper showed how to generate the Euler's use of series in the enumeration of partitions. In 1952, Macmahon also quoted the self-conjuate partitions of $n$. In this Paper, Macmahon's self-conjugate partitions are explained with the help of array of dots. This paper showed how to prove the Euler's Theorems with the help of Euler's device of the introduction of a second parameter z, and showed how to prove the Theorem 3 with the help of Euler's generating function for P(n), and also showed how to prove the Theorem 4 with the help of certain conditions of $\mathrm{P}(\mathrm{n})$.
\end{abstract}

Keywords: at most, array, convenient, discover, denominator, diagonal, Euler's device, Euler's identity, Macmahon's table, Self-conjugate

Cite This Article: Sabuj Das, “Some Restricted Partition Functions." American Journal of Applied Mathematics and Statistics, vol. 2, no. 6 (2014): 386-393. doi: 10.12691/ajams-2-6-5.

\section{Introduction}

In this paper, we give some related definitions of $\mathrm{P}(\mathrm{n})$, $\mathrm{P}^{\mathrm{e}}(\mathrm{n}), \mathrm{Q}^{\mathrm{o}}(\mathrm{n}), \mathrm{Q}^{\mathrm{e}}(\mathrm{n}), \mathrm{X}(\mathrm{n}), \mathrm{Y}(\mathrm{n}), \mathrm{P}(\mathrm{n}, \mathrm{p}, *), Q_{m}^{e}(n), Q_{m}^{o}$, $\sigma(n), S_{2 m}(n-m(m+l)), P(n, p, \leq q), P(n, \leq p, \leq q), P(n, \leq p$, $*)$, and establish the Remark 1: $\mathrm{X}(\mathrm{n})=\mathrm{Y}$ (n) with numerical example when $n=8$, and also formulate the generating functions

for

$P^{e}(n), X(n), Y(n), Q^{e}(n), Q^{o}(n), S_{2 m}(n-m(m+1))$

and $Q_{m}^{e}(n)-Q_{m}^{o}(n)$

We prove the Euler's Theorems with the help of Euler's device of the introduction of a second parameter $z$, when $\mathrm{z}=1$ and $\mathrm{z}=\mathrm{x}$ respectively, and describe elaborately all selfconjugate partitions of $n$ [5].We prove the Theorem 3 in terms of $\sigma(n)$, and prove the Theorem 4 with numerical example when $n=11$.

\section{Some Related Definitions}

$P(n)$ [3]: The number of partitions of n. Example: 4, $3+1,2+2,2+1+1,1+1+1+1 \therefore P(4)=5$.

$\mathrm{P}^{\mathrm{e}}(\mathrm{n})$ : The number of partitions of $\mathrm{n}$ into even parts.

$\mathrm{Q}^{\circ}(\mathrm{n})$ [6]: The number of partitions of $\mathrm{n}$ into distinct odd parts.
$Q^{\mathrm{e}}(\mathrm{n})$ : The number of partitions of $\mathrm{n}$ into distinct even parts.

$\mathrm{X}(\mathrm{n})$ [2]: The number of partitions of $\mathrm{n}$ with no part repeated more than twice.

$\mathrm{Y}(\mathrm{n})$ : The number of partitions of $\mathrm{n}$ with no part divisible by 3.

$\mathrm{P}\left(\mathrm{n}, \mathrm{p},{ }^{*}\right)$ : The number of partitions of $\mathrm{n}$ into $\mathrm{p}$ parts.

$\sigma(n)$ [4]: The sum of divisors of $n$ including 1 and $n$.

$Q_{m}^{e}(n)$ : The number of partitions of $n$ into an even number of distinct parts no greater than $\mathrm{m}$.

$Q_{m}^{o}$ : The number of partitions of $n$ into an odd number of distinct parts no greater than $\mathrm{m}$.

$\mathrm{S}_{2 \mathrm{~m}}(\mathrm{n}-\mathrm{m}(\mathrm{m}+1))$ : The number of partitions of $n-m(m+1)$ into even parts not exceeding $2 \mathrm{~m}$.

$\mathrm{P}(\mathrm{n}, \mathrm{p}, \leq \mathrm{q})$ : The number of partitions of $\mathrm{n}$ into $\mathrm{p}$ parts, none of which exceeds $\mathrm{q}$.

$\mathrm{P}(\mathrm{n}, \leq \mathrm{p}, \leq \mathrm{q})$ : The number of partitions of $\mathrm{n}$ into $\mathrm{p}$ or any smaller number of parts, none of which exceeds $\mathrm{q}$.

$\mathrm{P}(\mathrm{n}, \leq \mathrm{p}, *)$ : The number of partitions of $\mathrm{n}$ into $\mathrm{p}$ or any smaller number of parts.

\section{Construction of a Table of Values of $\mathbf{P}(\mathbf{n}, \mathbf{p}, *)$ ([1] and [5])}

Table of p-partitions of n (i.e. values of P(n, p, *).

\begin{tabular}{|c|c|c|c|c|c|c|c|c|c|c|c|c|c|c|c|c|c|}
\hline $\mathrm{n}=$ & 1 & 2 & 3 & 4 & 5 & 6 & 7 & 8 & 9 & 10 & 11 & 12 & 13 & 14 & 15 & 16 & 17 \\
\hline $\mathrm{p}=1$ & 1 & 1 & 1 & 1 & 1 & 1 & 1 & 1 & 1 & 1 & 1 & 1 & 1 & 1 & 1 & 1 & 1 \\
\hline 2 & & 1 & 1 & 2 & 2 & 3 & 3 & 4 & 4 & 5 & 5 & 6 & 6 & 7 & 7 & 8 & 8 \\
\hline 3 & & & 1 & 1 & 2 & 3 & 4 & 5 & 7 & 8 & 10 & 12 & 14 & 16 & 19 & 21 & 24 \\
\hline 4 & & & & 1 & 1 & 2 & 3 & 5 & 6 & 9 & 11 & 15 & 18 & 23 & 27 & 34 & 39 \\
\hline 5 & & & & & 1 & 1 & 2 & 3 & 5 & 7 & 10 & 13 & 18 & 23 & 30 & 37 & 47 \\
\hline
\end{tabular}




\begin{tabular}{|c|c|c|c|c|c|c|c|c|c|c|c|c|c|c|c|c|c|}
\hline 6 & & & & & & 1 & 1 & 2 & 3 & 5 & 7 & 11 & 14 & 20 & 26 & 35 & 44 \\
\hline 7 & & & & & & & 1 & 1 & 2 & 3 & 5 & 7 & 11 & 15 & 21 & 28 & 38 \\
\hline 8 & & & & & & & & 1 & 1 & 2 & 3 & 5 & 7 & 11 & 15 & 22 & 29 \\
\hline 9 & & & & & & & & & 1 & 1 & 2 & 3 & 5 & 7 & 11 & 15 & 22 \\
\hline 10 & & & & & & & & & & 1 & 1 & 2 & 3 & 5 & 7 & 11 & 15 \\
\hline 11 & & & & & & & & & & & 1 & 1 & 2 & 3 & 5 & 7 & 11 \\
\hline 12 & & & & & & & & & & & & 1 & 1 & 2 & 3 & 5 & 7 \\
\hline 13 & & & & & & & & & & & & & 1 & 1 & 2 & 3 & 5 \\
\hline 14 & & & & & & & & & & & & & & 1 & 1 & 2 & 3 \\
\hline 15 & & & & & & & & & & & & & & & 1 & 1 & 2 \\
\hline 16 & & & & & & & & & & & & & & & & 1 & 1 \\
\hline 17 & & & & & & & & & & & & & & & & & 1 \\
\hline Total & 1 & 2 & 3 & 5 & 7 & 11 & 15 & 22 & 30 & 42 & 56 & 77 & 101 & 135 & 176 & 231 & 297 \\
\hline
\end{tabular}

We get $\mathrm{P}(\mathrm{n}, 1, *)=1, \mathrm{P}(\mathrm{n}, 2, *)=\frac{1}{2}(\mathrm{n}-1)$ or $\frac{n}{2}$, when $\mathrm{n}$ is odd or even Also $\mathrm{P}(\mathrm{n}, \mathrm{n}, *)=1 \mathrm{P}(\mathrm{n}, \mathrm{n}-1 *)=1$. From above table; (n, k) is denoted by the n-th space of kth row, and the set of spaces $(n, 1),(n+1,2),(n+2,3),(n+3,4)$ ------- the $n$th diagonal.

We first complete the first and second rows and the first and second diagonals.

The integers of the $(n+1)$ th diagonal are the sums of one, two, three, four, ------ terms of the $n$th column, starting from the top.

That is, the second column is $1,10,0$----, so that the third diagonal is $1,2,2,2,2,--------$. The third column is $1,1,1,0,0,0$, ----, so that the fourth diagonal is $1,2,3,3$,

The fourth column is $1,2,1,1,0$, -------, so that the fifth diagonal is $1,3,4,5,5,5,5$, -----, i. e. the fifth diagonal is $1,1+2,1+2+1,1+2+1+1,1+2+1+1$ +0 , -----, and so on to any extent.

\section{We Discuss now about the Generating Functions for $\mathbf{P}^{\mathbf{e}}(\mathbf{n}), X(n), \mathbf{Y}(\mathbf{n}), \mathbf{Q}^{\mathbf{0}}(\mathbf{n})$, $\mathbf{Q}^{\mathrm{e}}(\mathbf{n}), \mathbf{S}_{2 \mathbf{m}}(\mathbf{n}-\mathbf{m}(\mathbf{m}+\mathbf{1}))$ and $Q_{m}^{e}(n)-Q_{m}^{o}$.} [These are Euler's use of series in the enumeration of partitions]

We consider a function, whose denominator is the product of infinite factors $\left(1-x^{2}\right),\left(1-x^{4}\right),------\infty$ and it is written as

$$
\begin{aligned}
& \frac{1}{\left(1-x^{2}\right)\left(1-x^{4}\right)\left(1-x^{6}\right)} \\
& =1+x^{2}+2 x^{4}+3 x^{6}+5 x^{8}+\cdots \infty \\
& =1+\sum_{n=1}^{\infty} P^{e}(n) x^{n}, . .
\end{aligned}
$$

where $\mathrm{P}^{\mathrm{e}}(\mathrm{n})$ is the number of partitions of $\mathrm{n}$ into even parts.

It is convenient to define $\mathrm{p}^{\mathrm{e}}(\mathrm{n})=0$, if $\mathrm{n}$ is an odd integer.

We consider a function, which is the product of infinite factors, one of which is $\left(1+x^{n}+x^{2 n}\right)$ and it can be written as

$$
\begin{aligned}
& \left(1+x+x^{2}\right)\left(1+x^{2}+x^{4}\right)\left(1+x^{3}+x^{6}\right) \ldots \infty \\
& =1+x+2 x^{2}+2 x^{3}+4 x^{4}+5 x^{5}+7 x^{6}+\cdots \infty \\
& =1+\sum_{n=1}^{\infty} X(n) x^{n}, \ldots
\end{aligned}
$$

Each element of the product comes from multiplying together one term from each bracket either $x^{0}$ or $x^{n}$ or $x^{n+n}$ from $1+x^{n}+x^{2 n}$. So in the corresponding partition no part occurs more than twice.

Therefore we can say that the coefficient $X(n)$ of $x^{\mathrm{n}}$ in the above expansion is the number of partitions of $n$ with no part repeated more than twice.

The generating function for $Y(n)$ is of the form

$$
\begin{aligned}
& \frac{1}{(1-x)\left(1-x^{2}\right)\left(1-x^{4}\right)\left(1-x^{5}\right) \ldots\left(1-x^{3 n-2}\right)\left(1-x^{3 n-1}\right) \ldots \infty} \\
& =1+x+2 x^{2}+2 x^{3}+4 x^{4}+5 x^{5}+7 x^{6}+\cdots \infty \\
& =1+\sum_{n=1}^{\infty} Y(n) x^{n}, \ldots
\end{aligned}
$$

where the coefficient $Y(n)$ is the number of partitions of $\mathrm{n}$ with no part divisible by 3 . From (2) and (3) we have

$$
\begin{aligned}
& 1+\sum_{n=1}^{\infty} X(n) x^{n} \\
& =\left(1+x+x^{2}\right)\left(1+x^{2}+x^{4}\right) \\
& \left(1+x^{3}+x^{6}\right) \ldots\left(1+x^{n}+x^{2 n}\right) \ldots \infty \\
& =\frac{1-x^{3}}{1-x} \cdot \frac{1-x^{6}}{1-x^{2}} \cdot \frac{1-x^{9}}{1-x^{3}} \cdots \frac{1-x^{3 n}}{1-x^{n}} \cdots \infty \\
& =\frac{1}{(1-x)\left(1-x^{2}\right)\left(1-x^{4}\right)\left(1-x^{5}\right) \ldots\left(1-x^{3 n-2}\right)\left(1-x^{3 n-1}\right) \ldots \infty} \\
& =1+\sum_{n=1}^{\infty} Y(n) x^{n} .
\end{aligned}
$$

Equating the coefficient of $\mathrm{x}^{\mathrm{n}}$ from both sides, we get the following remark.

Remark 1[8]: $X(n)=Y(n)$, i.e. the number of partitions of $n$ with no part repeated more

than twice is equal to the number of partitions of $n$ with no part divisible by 3 .

Proof: We develop a one-to-one correspondence between the partitions enumerated by $X(n)$ and those enumerated by $Y(n)$. Let $n=a_{1}+a_{2}+\ldots+a_{r}$ be a partition of $n$ with no part is repeated more than twice. We transfer this into a partition of $n$ with no part is divisible by 3. If a part $a_{m}$ of the partition, which is divisible by 3 , enumerated by $X(n)$ can be expressed into three equal parts, such that: $6=2+2+2,3=1+1+1$. Rearranging the 
parts of the partition, we can say that the parts are not divisible by 3. Clearly, our correspondence is one-to-one.

Conversely, we start any partition of $n$ into with no part is divisible by 3 , say $n=a_{1}+a_{2}+\ldots+a_{r}$, we consider the same part not less than thrice, it would be unique sum by same three parts by taking a group, such that, $5+1+1+1=$ $5+3$ and $2+2+2+1+1=6+1+1$.

This gives $n$ as a partition with no part is repeated more than twice. Thus, we have the one-to-one correspondence. The corresponding is onto, so that $X(n)=Y(n)$. Hence the Theorem.

Example 1: When $n=8$, the listed partitions of 8 with no part repeated more than twice is given below:

$8=7+1=6+2=6+1+1=5+3=5+2+1=4+4=5+3+1$ $=4+2+1+1=4+2+2=3+3+2=3+3+1+1=3+2+2+1$. So, there are 13 partitions i.e., $X(8)=13$. Again, the list of partitions of 8 with no part is divisible by 3 is given below: $8=7+1=5+2+1=5+1+1+1+1=4+4=4+2+1+1=$ $4+2+2=4+1+1+1+1=2+2+2+2=2+2+2+1+1=$ $2+2+1+1+1+1=2+1+1+1+1+1+1=1+1+1+1+1+1+1+1$.

So, there are 13 partitions i.e., $Y(8)=13$. $\therefore X(n)=Y(n)$.

Here we formulate the generating function[3], which enumerates all self-conjugate partitions of $\mathbf{n}$.

We can represent the partition $15+5+1$ of 21 graphically by an array of dots as in $\mathrm{A}_{1}$

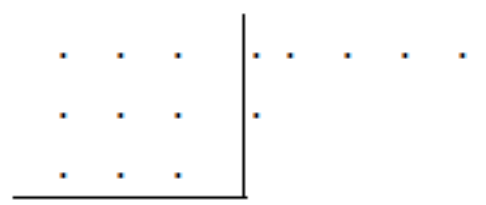

$\mathrm{A}_{1}$

Here we have a square of $3^{2}$ points and two tails, each representing a partition of $\frac{1}{2}\left(21-3^{2}\right)=\frac{1}{2} \cdot 12=6$ into 3 parts at most.

Generally a self-conjugate partition of $\mathrm{n}$ can be read as a square of $\mathrm{m}^{2}$ dots and two tails representing partitions of $\frac{1}{2}\left(n-m^{2}\right)$ into m parts at most.

So we can say that $\frac{x^{m^{2}}}{\left(1-x^{2}\right)\left(1-x^{4}\right) \ldots\left(1-x^{2 m}\right)}$ enumerates the partitions of $n-m^{2}$ into even parts not exceeding $2 m$ or of $\frac{1}{2}$ (n-m ${ }^{2}$ ) into parts not exceeding $m$ or of $\frac{1}{2}\left(n-m^{2}\right)$ into at most m parts. Now each of these corresponds represents a self-conjugate partition of $n$ having a square of $\mathrm{m}^{2}$ points. Hence we have $1+\sum_{m=1}^{\infty} \frac{x^{m^{2}}}{\left(1-x^{2}\right)\left(1-x^{4}\right) \ldots\left(1-x^{2 m}\right)}$ enumerates all selfconjugate partitions of $n$. Thus for $n=10: 7+3=9+1$ has two partitions, which have distinct odd parts. These two partitions are self-conjugate partitions and their graphs are as follows

The generating function for $S_{2 m}(n-m(m+1))$ is of the form

$$
\begin{aligned}
& \frac{x^{m(m+1)}}{\left(1-x^{2}\right)\left(1-x^{4}\right) \ldots\left(1-x^{2 m}\right)} \\
& =x^{m(m+1)} \sum_{n=m(m+1)}^{\infty} S_{2 m}(n-m(m+1)) x^{m(m+1)} \\
& =\sum_{n=m(m+1)}^{\infty} S_{2 m}(n-m(m+1)) x^{n}, \ldots
\end{aligned}
$$

where the coefficient $S_{2 m}(n-m(m+1))$ is the number of partitions of $n-m(m+1)$ into even parts not exceeding $2 m$ or of $\frac{1}{2}(n-m(m+1))$ into parts not exceeding $m$.

Example 2: If $\mathrm{m}=3$ the above expansion becomes;

$$
\begin{aligned}
& \frac{x^{12}}{\left(1-x^{2}\right)\left(1-x^{4}\right)\left(1-x^{6}\right)} \\
& =x^{12}\left\{1+x^{2}+2 x^{4}+3 x^{6}+4 x^{8}+\ldots \infty\right\} \\
& =\sum_{n=12}^{\infty} S_{6}(n-12) x^{n}
\end{aligned}
$$

It is convenient to define $S_{6}(0)=1$ and $S_{6}(n)=0$, if $n$ is odd integer.

The generating function for $\mathrm{Q}^{\circ}(\mathrm{n})$ is of the form

$$
\begin{aligned}
& (1+x)\left(1+x^{3}\right)\left(1+x^{5}\right) \ldots \infty \\
& =1+x+x^{2}+x^{3}+x^{4}+x^{5}+x^{6}+x^{7}+2 x^{8}+\ldots \infty \\
& =1+\sum_{n=1}^{\infty} Q^{o}(n) x^{n}, . .
\end{aligned}
$$

where the coefficient $Q^{\circ}(n)$ is the number of partitions of $n$ into distinct odd parts.

The generating function for $\mathrm{Q}^{\mathrm{e}}(\mathrm{n})$ is of the form

$$
\begin{aligned}
& \left(1+x^{2}\right)\left(1+x^{4}\right)\left(1+x^{6}\right)\left(1+x^{8}\right) \ldots \infty \\
& =1+x^{2}+x^{4}+2 x^{6}+2 x^{8}+3 x^{10}+4 x^{12}+\ldots \infty \\
& =1+\sum_{n=1}^{\infty} Q^{e}(n) x^{n}, . .
\end{aligned}
$$


It is convenient to define $\mathrm{Q}^{\mathrm{e}}(0)=1$ and $\mathrm{Q}^{\mathrm{e}}(\mathrm{n})=0$, if $\mathrm{n}$ is odd positive integer.

The coefficient $Q^{e}(n)$ of $x^{n}$ in the above expansion is the number of partitions of $n$ into distinct even parts.

From above expansions we get the following remarks.

[These are known as Euler's Theorems]

Theorem 1:

$$
\begin{aligned}
& (1+x)\left(1+x^{3}\right)\left(1+x^{5}\right) \ldots \infty \\
& =1+\frac{x}{1-x^{2}}+\frac{x^{4}}{\left(1-x^{2}\right)\left(1-x^{4}\right)}+\ldots \infty
\end{aligned}
$$

i.e.

$$
\begin{aligned}
& (1+x)\left(1+x^{3}\right)\left(1+x^{5}\right) \ldots \infty \\
& =1+\sum_{m=1}^{\infty} \frac{x^{m^{2}}}{\left(1-x^{2}\right)\left(1-x^{4}\right) \ldots\left(1-x^{2 m}\right)}
\end{aligned}
$$

Theorem 2:

$$
\begin{aligned}
& \left(1+x^{2}\right)\left(1+x^{4}\right)\left(1+x^{6}\right) \ldots \infty \\
& =1+\frac{x^{2}}{1-x^{2}}+\frac{x^{6}}{\left(1-x^{2}\right)\left(1-x^{4}\right)}+\ldots \infty
\end{aligned}
$$

i.e,

$$
\begin{aligned}
& \left(1+x^{2}\right)\left(1+x^{4}\right)\left(1+x^{6}\right) \ldots \infty \\
& =1+\sum_{m=1}^{\infty} \frac{x^{m(m+1)}}{\left(1-x^{2}\right)\left(1-x^{4}\right) \ldots\left(1-x^{2 m}\right)} \ldots
\end{aligned}
$$

\section{Proof of Remarks:}

We have

$$
\begin{gathered}
\left(1-x^{2}\right)^{-1}=1+x^{2}+x^{4}+x^{6}+x^{8}+\ldots \infty, . . \\
\left(1-x^{2}\right)^{-1}\left(1-x^{4}\right)^{-1}=1+x^{2}+2 x^{4}+2 x^{6}+2 x^{8}+\ldots \infty, . . \\
\left(1-x^{2}\right)^{-1}\left(1-x^{4}\right)^{-1}\left(1-x^{6}\right)^{-1} \\
=1+x^{2}+2 x^{4}+3 x^{6}+4 x^{8}+. . \infty
\end{gathered}
$$

and so on.

These are used in proving the Theorems.

Now we shall convert the function into the another function. We have the function

$$
(1+z x)\left(1+z x^{3}\right)\left(1+z x^{5}\right)\left(1+z x^{7}\right) \ldots \infty
$$

(where $\mathrm{z} \in(-1,1]$ and $|x|<1$ )

$$
\begin{aligned}
& =\left(\begin{array}{c}
1+z\left(x+x^{3}+x^{5}\right) \\
+z^{2}\left(x^{4}+x^{6}+x^{8}\right)+z^{3} x^{9}
\end{array}\right)\left(1+z x^{7}\right)\left(1+z x^{9}\right) \ldots \infty \\
& =1+z x\left(1+x^{2}+x^{4}+x^{6}+\ldots \infty\right) \\
& +z^{2} x^{4}\left(1+x^{2}+2 x^{4}+2 x^{6}+3 x^{8}+\ldots \infty\right) \\
& +z^{3} x^{9}\left(1+x^{2}+2 x^{4}+3 x^{6}+4 x^{8}+\ldots \infty\right)+\ldots \infty
\end{aligned}
$$

$$
\begin{aligned}
& =1+z x\left(1-x^{2}\right)^{-1}+z^{2} x^{4}\left(1-x^{2}\right)^{-1}\left(1-x^{4}\right)^{-1} \\
& +z^{3} x^{9}\left(1-x^{2}\right)^{-1}\left(1-x^{4}\right)^{-1}\left(1-x^{6}\right)^{-1}+\ldots \infty
\end{aligned}
$$$$
\text { (by (9),(10),(11)) }
$$$$
=1+\frac{z x}{\left(1-x^{2}\right)}+\frac{z^{2} x^{4}}{\left(1-x^{2}\right)\left(1-x^{4}\right)}
$$$$
+\frac{z^{3} x^{9}}{\left(1-x^{2}\right)\left(1-x^{4}\right)\left(1-x^{6}\right)}+\ldots+\infty
$$

If $\mathrm{z}=1$, then (12) becomes

$$
\begin{gathered}
\quad(1+x)\left(1+x^{3}\right)\left(1+x^{5}\right) \ldots \infty \\
=1+\frac{x}{\left(1-x^{2}\right)}+\frac{x^{4}}{\left(1-x^{2}\right)\left(1-x^{4}\right)} \\
+\frac{x^{9}}{\left(1-x^{2}\right)\left(1-x^{4}\right)\left(1-x^{6}\right)}+\ldots+\infty \\
(1+x)\left(1+x^{3}\right)\left(1+x^{5}\right) \ldots \infty \\
\text { i.e. }=1+\sum_{m=1}^{\infty} \frac{x^{m^{2}}}{\left(1-x^{2}\right)\left(1-x^{4}\right) \ldots\left(1-x^{2 m}\right)} \ldots
\end{gathered}
$$

Hence the Theorem.

If $\mathrm{z}=\mathrm{x}$, then (12) becomes;

$$
\begin{aligned}
& \left(1+x^{2}\right)\left(1+x^{4}\right)\left(1+x^{6}\right) \ldots \infty \\
& =1+\frac{x^{2}}{\left(1-x^{2}\right)}+\frac{x^{6}}{\left(1-x^{2}\right)\left(1-x^{4}\right)} \\
& +\frac{x^{12}}{\left(1-x^{2}\right)\left(1-x^{4}\right)\left(1-x^{6}\right)}+\ldots+\infty
\end{aligned}
$$

where the indices in the numerators

$1.2,2.3,3.4, \ldots \ldots$.

$$
\begin{aligned}
& \left(1+x^{2}\right)\left(1+x^{4}\right)\left(1+x^{6}\right) \ldots \infty \\
\text { i.e. } & =1+\sum_{m=1}^{\infty} \frac{x^{m(m+1)}}{\left(1-x^{2}\right)\left(1-x^{4}\right) \ldots\left(1-x^{2 m}\right)} \ldots
\end{aligned}
$$

Theorem.

We also get that Theorem 1 represents the following statement.

The number of partitions of $\mathrm{n}$ into odd and unequal parts is equal to the numbers of it self-conjugate partitions.

We consider a function $\varphi_{m}(x)$, which is the product of $\mathrm{m}$ factors

$(1-\mathrm{x}),\left(1-\mathrm{x}^{2}\right),-----\left(1-\mathrm{x}^{\mathrm{m}}\right)$ as in the form $\varphi_{m}(x)=(1-\mathrm{x})(1-$ $\left.x^{2}\right)\left(1-x^{3}\right)--\left(1-x^{m}\right)$

$$
\frac{m(m+1)}{2}=\sum_{n=0}^{2}\left(Q_{m}^{e}(n)-Q_{m}^{o}(n)\right) x^{n} .
$$

It is convenient to define $Q_{m}^{e}(0)=1, Q_{m}^{o}(0)=0$.

Example 3: If $\mathrm{m}=4$, the above expansion becomes 


$$
\begin{aligned}
& \varphi_{4}(x)=(1-\mathrm{x})\left(1-\mathrm{x}^{2}\right)\left(1-\mathrm{x}^{3}\right)\left(1-\mathrm{x}^{4}\right) \\
& =1-\mathrm{x}-\mathrm{x}^{2}+2 \mathrm{x}^{5}-\mathrm{x}^{8}-\mathrm{x}^{9}+\mathrm{x}^{10} \\
& =\sum_{n=0}^{10}\left(Q_{4}^{e}(n)-Q_{4}^{o}(n)\right) x^{n} \\
& =1+\sum_{n=0}^{10}\left(Q_{4}^{e}(n)-Q_{4}^{o}(n)\right) x^{n}
\end{aligned}
$$

Since $Q_{4}^{e}(1)=0, Q_{4}^{o}(1)=1$ with relevant partitions is 1. So $Q_{4}^{e}(1)-Q_{4}^{o}(1)=-1$

$Q_{4}^{e}(2)=0, Q_{4}^{o}(2)=1$ with relevant partition is 2 , so $Q_{4}^{e}(2)-Q_{4}^{o}(2)=-1$

$Q_{4}^{e}(3)=1$ with relevant partition is $2+1, Q_{4}^{o}(3)=1$ with relevant partition is 3 , So $Q_{4}^{e}(3)-Q_{4}^{o}(3)=1-1=0$.

$Q_{4}^{e}(4)=1$ with relevant partition is $3+1$ and $Q_{4}^{o}(4)=$ 1 with relevant partition is 4 , so $Q_{4}^{e}(4)-Q_{4}^{o}(4)=1-1=0$.

$Q_{4}^{e}(5)=2$ with relevant partitions being $4+1,3+2$ and $Q_{4}^{o}(5)=0, Q_{4}^{e}(5)-Q_{4}^{o}(5)=2$.

$Q_{4}^{e}(6)=1$ with relevant partition is $4+2$ and $Q_{4}^{o}(6)=1$ with relevant partition is $3+2+1$, so $Q_{4}^{e}(6)-Q_{4}^{o}(6)=1-1$ $=0$.

$Q_{4}^{e}(7)=1$ with relevant partition is $4+3$ and $Q_{4}^{o}(7)=1$ with relevant partition is $4+2+1$, so $Q_{4}^{e}(7)-Q_{4}^{o}(7)=1$ $-1=0$.

$Q_{4}^{e}(8)=0$ and $Q_{4}^{o}(8)=1$ with relevant partition is 4+3+1, so $Q_{4}^{e}(8)-Q_{4}^{o}(8)=0-1=-1$.

$Q_{4}^{e}(9)=0$ and $Q_{4}^{o}(9)=1$ with relevant partition is $4+3+2$, so $Q_{4}^{e}(9)-Q_{4}^{o}(9)=0-1=-1$.

$Q_{4}^{e}(10)=1$ with relevant partition is $4+3+2+1$, and $Q_{4}^{o}(10)=0$, so $Q_{4}^{e}(10)-Q_{4}^{o}(10)=1-0=1$.

Here we give the following Theorem, which is related to the term $\sigma(\mathrm{n})$.

Theorem 3: If $\mathrm{n}$ is any positive integer, then $\mathrm{nP}(\mathrm{n})=$ $\mathrm{P}(0) \sigma(\mathrm{n})+\mathrm{P}(1) \sigma(\mathrm{n}-1)+---+\mathrm{P}(\mathrm{r}) \sigma(\mathrm{n}-\mathrm{r})+---+\mathrm{P}(\mathrm{n}-1)$ $\sigma(1)$.

Proof: We have the generating function for $\mathrm{P}(\mathrm{n})$ is

$$
\begin{aligned}
& f(x)=\frac{1}{(1-x)\left(1-x^{2}\right)\left(1-x^{3}\right) \cdots \infty} \\
& =\sum_{n=0}^{\infty} P(n) x^{n}, . .
\end{aligned}
$$

If it gives $\mathrm{f}(\mathrm{x})=\frac{1}{\varphi(x)}, \ldots$.

where $\varphi(x)=(1-x)\left(1-x^{2}\right)\left(1-x^{3}\right) \ldots \infty$

or, $\log \varphi(x)=\log (1-x)+\log \left(1-x^{2}\right)+\log \left(1-x^{3}\right)+\ldots \infty$

$=\sum_{n=1}^{\infty} \log \left(1-x^{k}\right)$

Differentiating it w. r. to $\mathrm{x}$ we get;

$$
\frac{\varphi^{\prime}(x)}{\varphi(x)}=\sum_{k=1}^{\infty} \frac{-k x^{k-1}}{1-x^{k}} \ldots
$$

From (14) we get; $f^{\prime}(x)=-\frac{\varphi^{\prime}(x)}{\varphi^{2}(x)}-\frac{\varphi^{\prime}(x)}{\varphi(x)} \cdot \frac{1}{\varphi(x)}$

or, $\sum_{n=1}^{\infty} P(n) n x^{n-1}=-\frac{\varphi^{\prime}(x)}{\varphi(x)} f(x)$ (by (13) and (14))

or, $\sum_{n=1}^{\infty} n P(n) n x^{n-1}=\sum_{k=1}^{\infty} \frac{k x^{k}}{1-x^{k}} \sum_{n=0}^{\infty} P(n) n x^{n} \quad$ (by

(15))

$=\left(\mathrm{P}(0)+\mathrm{P}(1) \mathrm{x}+\mathrm{P}(2) \mathrm{x}^{2}+\cdots \infty\right)$

$\sum_{k=1}^{\infty} \mathrm{k}\left(\mathrm{x}^{\mathrm{k}}+\mathrm{x}^{2 \mathrm{k}}+\mathrm{x}^{3 \mathrm{k}}+\cdots \infty\right)=$

$P(0)\left[\begin{array}{l}\left(x+x^{2}+x^{3}+x^{4}+\cdots \infty\right) \\ +2\left(x^{2}+x^{4}+\cdots \infty\right)+3\left(x^{3}+x^{6}+\cdots \infty\right) \\ +4\left(x^{4}+\cdots \infty\right)+\cdots\end{array}\right]$

$+\mathrm{P}(1)\left[\begin{array}{l}\left(\mathrm{x}^{2}+\mathrm{x}^{3}+\mathrm{x}^{4}+\cdots \infty\right)+2\left(\mathrm{x}^{3}+\mathrm{x}^{5}+\cdots \infty\right) \\ +3\left(\mathrm{x}^{4}+\mathrm{x}^{7}+\cdots \infty\right)+4\left(\mathrm{x}^{5}+\cdots \infty\right)+\cdots\end{array}\right]$

$+\mathrm{P}(2)\left[\left(\mathrm{x}^{3}+\mathrm{x}^{4}+\cdots \infty\right)+2\left(\mathrm{x}^{4}+\cdots \infty\right)+\cdots \infty\right]$

$+\mathrm{P}(3)\left[\left(\mathrm{x}^{4}+\cdots \infty\right)+2\left(\mathrm{x}^{5}+\cdots \infty\right)+\cdots \infty\right]$

$+P(4)\left[\begin{array}{l}\left(x^{5}+x^{6}+\cdots \infty\right)+2\left(x^{6}+x^{8}+\cdots \infty\right) \\ +3\left(x^{7}+x^{10}+\cdots \infty\right)+4\left(x^{8}+\cdots \infty\right) \\ +\cdots \infty\end{array}\right]$

$+\cdots \infty$.

$=\mathrm{xP}(0)+\mathrm{x}^{2}(\mathrm{P}(0)+2 \mathrm{P}(0)+\mathrm{P}(1))$

$+\mathrm{x}^{3}(\mathrm{P}(0)+3 \mathrm{P}(0)+\mathrm{P}(1)+2 \mathrm{P}(1)+\mathrm{P}(2))$

$+\mathrm{x}^{4}\left(\begin{array}{l}\mathrm{P}(0)+2 \mathrm{P}(0)+4 \mathrm{P}(0)+\mathrm{P}(1) \\ +3 \mathrm{P}(1)+\mathrm{P}(2)+2 \mathrm{P}(2)+\mathrm{P}(3)\end{array}\right)+\cdots \infty$

$=\sum_{n=1}^{\infty} \mathrm{x}^{\mathrm{n}} \sum_{m=0}^{n-1} \mathrm{P}(\mathrm{m})\left(\sum_{k \mid n-m} k\right)$

$=\sum_{n=1}^{\infty} \mathrm{x}^{\mathrm{n}} \sum_{m=0}^{n-1} \mathrm{P}(\mathrm{m}) \sigma(n-m)$

Now equating the coefficients of $\mathrm{x}^{\mathrm{n}}$ from both sides, we $\mathrm{nP}(\mathrm{n})=\sum_{m=0}^{n-1} \mathrm{P}(\mathrm{m}) \sigma(\mathrm{n}-\mathrm{m})$, i. e. $\mathrm{nP}(\mathrm{n})$

get; $=\mathrm{P}(0) \mathrm{s}(\mathrm{n})+\mathrm{P}(1) \mathrm{s}(\mathrm{n}-1)+\ldots+\mathrm{P}(\mathrm{r}) \mathrm{s}(\mathrm{n}-\mathrm{r})$ Hence $+\ldots+\mathrm{P}(\mathrm{n}-1) \mathrm{s}(1)$.

the Theorem. 


\section{We also Discuss about the Restricted Partition Functions [7]. [These are also Euler's use of series in the enumeration of partitions]}

The generating function for $\mathrm{P}(\mathrm{n}, \mathrm{p}, \leq \mathrm{q})$ is of the form.

$$
\begin{aligned}
& \frac{1}{(1-z x)\left(1-z x^{2}\right) \cdots\left(1-z x^{q}\right)} \\
& =1+\sum_{n=1}^{\infty} x^{n}\left\{\sum_{p=1}^{n} z^{p} P(n, p \leq q)\right\} \cdots
\end{aligned}
$$

It is convenient to define $\mathrm{P}(\mathrm{n}, \mathrm{p}, \leq \mathrm{q})=0$, if $\mathrm{n}<\mathrm{p}$.

The coefficient $P(n, p, \leq q)$ is the number of partitions of $\mathrm{n}$ into $\mathrm{p}$ parts, none of which exceeds $\mathrm{q}$.

Example 4: If $q=3$, the above function becomes

$$
\begin{aligned}
& \frac{1}{(1-z x)\left(1-z x^{2}\right)\left(1-z x^{3}\right)} \\
& =1+z x+x^{2}\left(z+z^{2}\right)+x^{3}\left(z+z^{2}+z^{3}\right) \\
& +x^{4}\left(2 z^{2}+z^{3}+z^{4}\right)+\cdots \infty \\
& =1+\sum_{n=1}^{\infty} x^{n}\left\{\sum_{p=1}^{n} z^{p} P(n, p \leq 3)\right\} .
\end{aligned}
$$

From (16) we have;

$$
\begin{aligned}
& 1+\sum_{n=1}^{\infty} x^{n}\left\{\sum_{p=1}^{n} z^{p} P(n, p \leq q)\right\} \\
& =\frac{1}{(1-z x)\left(1-z x^{2}\right) \cdots\left(1-z x^{q}\right)} \\
& =1+\sum_{n=1}^{\infty} x^{n}\left\{\sum_{p=1}^{n} z^{p} P(n, \leq q, p)\right\} .
\end{aligned}
$$

Equating the coefficient of $\mathrm{x}^{\mathrm{n}} \mathrm{z}^{\mathrm{p}}$ from both sides, we get the following remarks.

Remark 2: $\mathrm{P}(\mathrm{n}, \mathrm{p}, \leq \mathrm{q})=\mathrm{P}(\mathrm{n}, \leq \mathrm{q}, \mathrm{p})$.

If $q \rightarrow \infty$ in (16), such as $\lim _{q \rightarrow \infty} x^{q}=0,|x|<1$ then (16) becomes;

$$
\begin{aligned}
& \frac{1}{(1-z x)\left(1-z x^{2}\right)\left(1-z x^{3}\right) \cdots \infty} \\
& =1+\mathrm{zx}+\mathrm{x}^{2}\left(\mathrm{z}+\mathrm{z}^{2}\right)+\mathrm{x}^{3}\left(\mathrm{z}+\mathrm{z}^{2}+\mathrm{z}^{3}\right) \\
& +\mathrm{x}^{4}\left(2 \mathrm{z}^{2}+\mathrm{z}^{3}+\mathrm{z}^{4}\right)+\cdots \infty \\
& =1+\sum_{n=1}^{\infty} x^{n}\left\{\sum_{p=1}^{n} z^{p} P(n, p, *)\right\} .
\end{aligned}
$$

where the coefficient $\mathrm{P}(\mathrm{n}, \mathrm{p}, *)$ is the number of partitions of $n$ into p parts.

From (17) we have;

$$
\begin{aligned}
& \frac{1}{(1-z x)\left(1-z x^{2}\right)\left(1-z x^{3}\right) \cdots \infty} \\
& =1+\mathrm{zx}+\mathrm{x}^{2}\left(\mathrm{z}+\mathrm{z}^{2}\right)+\mathrm{x}^{3}\left(\mathrm{z}+\mathrm{z}^{2}+\mathrm{z}^{3}\right) \\
& +\mathrm{x}^{4}\left(2 \mathrm{z}^{2}+\mathrm{z}^{3}+\mathrm{z}^{4}\right)+\cdots \infty \\
& =1+\sum_{n=1}^{\infty} x^{n}\left\{\sum_{p=1}^{n} z^{p} P\left(n,{ }^{*}, p\right)\right\} .
\end{aligned}
$$

Equating the coefficient of $\mathrm{x}^{\mathrm{n}} \mathrm{z}^{\mathrm{p}}$ from both sides, we get the following remark.

Remark 3: $\mathrm{P}\left(\mathrm{n}, \mathrm{p},{ }^{*}\right)=\mathrm{P}(\mathrm{n}, *, \mathrm{p})$.

Proof of Remark 3: We can represent a partition of 15 graphically by an array of dots or nodes such as

The dots in a column corresponding to a part. Thus B represents the partition $6+4+3+1+1$ of 15 . We can also represent $\mathrm{B}$ by transposing rows and columns in which case it would represent the partition graphically as conjugate of $\mathrm{B}$

\section{Conjugate of $B$}

The dots in a column corresponding to a part, so that it represents the partition $5+3+3+2+1+1$ of 15 . Such pair of partitions are said to be conjugate. The largest part in either of these partitions is equal to the number of parts in the other. So that we can say that the number of partitions of $n$ into $p$ parts is the same as the number of partitions of $\mathrm{n}$ having largest part $\mathrm{p}$, i. e. $\mathrm{P}\left(\mathrm{n}, \mathrm{p},{ }^{*}\right)=\mathrm{P}\left(\mathrm{n},{ }^{*}, \mathrm{p}\right)$. Hence the Remark.

Example 5: The list of partitions of 8 into 4 parts is given below

$5+1+1+1=4+2+1+1=3+3+1+1=3+2+2$ $+1=2+2+2+2$

The number of such partitions is 5 , i.e. $\mathrm{P}(8,4, *)=5$.

Again the list of partitions of 8 having largest part 4 is given below

$4+4=4+3+1=4+2+1+1=4+1+1+1+1=4$ $+2+2$.

So the number of such partitions is 5 . i.e. $\mathrm{P}(8, *, 4)=5$.

Here $4+4,4+3+1,4+2+1+1,4+1+1+1+1$, and $4+2+2$ are the conjugate partitions of $2+2+2+2$, $3+2+2+1,4+2+1+1,5+1+1+1$ and $3+3+1+$ 1 respectively. Thus the number of partitions of 8 into 4 parts is equal to the number of partitions of 8 into parts, the largest of which is 4 .

Generally, we can say that the number of partitions of $n$ into $p$ parts is the same as the number of partitions of $n$ having largest part $\mathrm{p}$. 
The generating function for $\mathrm{P}(\mathrm{n}, \leq \mathrm{p}, \leq \mathrm{q})$ is of the form

$$
\begin{aligned}
& \frac{1}{(1-z x)\left(1-z x^{2}\right) \cdots\left(1-z x^{q}\right)} \\
& =1+\sum_{n=1}^{\infty} x^{n}\left\{\sum_{p=1}^{n} z^{p} P(n, \leq p, \leq q)\right\} \cdots
\end{aligned}
$$

where the coefficient $\mathrm{P}(\mathrm{n}, \leq \mathrm{p}, \leq \mathrm{q})$ is the number of partitions of $n$ into $p$ or any

smaller number of parts, none of which exceeds q.

Example 6: If $q=2$ then the function (18) becomes

$$
\begin{aligned}
& \frac{1}{(1-z)(1-z x)\left(1-z x^{2}\right)} \\
& =1+\left(\mathrm{z}+\mathrm{z}^{2}+\mathrm{z}^{3}+\cdots \infty\right)+\mathrm{x}\left(\mathrm{z}+\mathrm{z}^{2}+\mathrm{z}^{3}+\cdots \infty\right) \\
& +\mathrm{x}^{2}\left(\mathrm{z}+2 \mathrm{z}^{2}+\cdots \infty\right)+\mathrm{x}^{3}\left(\mathrm{z}^{2}+2 \mathrm{z}^{3}+\cdots \infty\right)+\cdots \infty \\
& =1+\sum_{n=0}^{\infty} x^{n}\left\{\sum_{p=1}^{n} \mathrm{z}^{p} P(n, \leq p, \leq 2)\right\}
\end{aligned}
$$

If $\mathrm{q} \rightarrow \infty$, such as $\quad \mathrm{x}^{\mathrm{q}}=0,|\mathrm{x}|<1$, then (18) becomes;

$$
\begin{aligned}
& \frac{1}{(1-z)(1-z x)\left(1-z x^{2}\right) \cdots \infty} \\
& =1+\left(\mathrm{z}+\mathrm{z}^{2}+\mathrm{z}^{3}+\cdots \infty\right)+\mathrm{x}\left(\mathrm{z}+\mathrm{z}^{2}+\mathrm{z}^{3}+\cdots \infty\right) \\
& +\mathrm{x}^{2}\left(\mathrm{z}+2 \mathrm{z}^{2}+\cdots \infty\right)+\cdots \infty \\
& =1+\sum_{n=0}^{\infty} x^{n}\left\{\sum_{p=1}^{n} z^{p} P(n, \leq p, *)\right\}
\end{aligned}
$$

where the coefficient $\mathrm{P}(\mathrm{n}, \leq \mathrm{p}, *)$ is the number of partitions of $\mathrm{n}$ into $\mathrm{p}$ or any smaller number of parts. From (19) We have;

$$
\begin{aligned}
& =1+\sum_{n=0}^{\infty} x^{n}\left\{\sum_{p=1}^{n} z^{p} P(n, \leq p, *)\right\} \\
& =1+\left(\mathrm{z}+\mathrm{z}^{2}+\mathrm{z}^{3}+\cdots \infty\right)+\mathrm{x}\left(\mathrm{z}+\mathrm{z}^{2}+\mathrm{z}^{3}+\cdots \infty\right) \\
& +\mathrm{x}^{2}\left(\mathrm{z}+2 \mathrm{z}^{2}+\cdots \infty\right)+\cdots \infty \\
& =1+\sum_{n=0}^{\infty} x^{n}\left\{\sum_{p=1}^{n} \mathrm{z}^{p} P(n+p, p, *)\right\}
\end{aligned}
$$

Equating the coefficient of $\mathrm{x}^{\mathrm{n}} \mathrm{z}^{\mathrm{p}}$ from both sides, we get the following remark.

Remark 4: $\mathrm{P}\left(\mathrm{n}, \leq \mathrm{p},{ }^{*}\right)=\mathrm{P}\left(\mathrm{n}+\mathrm{p}, \mathrm{p},{ }^{*}\right)$

Again from (19) we have;

$$
\begin{aligned}
& 1+\sum_{n=0}^{\infty} x^{n}\left\{\sum_{p=1}^{n} z^{p} P(n, \leq p, *)\right\} \\
& =1+\left(\mathrm{z}+\mathrm{z}^{2}+\mathrm{z}^{3}+\cdots \infty\right)+\mathrm{x}\left(\mathrm{z}+\mathrm{z}^{2} \cdots \infty\right) \\
& +\mathrm{x}^{2}\left(\mathrm{z}+2 \mathrm{z}^{2}+\cdots \infty\right)+\mathrm{x}^{3}(\mathrm{z}+\cdots \infty)+\cdots \infty \\
& =1+\sum_{n=0}^{\infty} x^{n}\left\{\sum_{p=1}^{n} \mathrm{z}^{p} P(n, *, \leq p)\right\}
\end{aligned}
$$

Equating the coefficient of $\mathrm{x}^{\mathrm{n}} \mathrm{z}^{\mathrm{p}}$ from both sides, we get the following remark.
Remark 5: $\mathrm{P}\left(\mathrm{n}, \leq \mathrm{p},{ }^{*}\right)=\mathrm{P}(\mathrm{n}, *, \leq \mathrm{p})$

Proof: The generating function for $\mathrm{P}_{\mathrm{p}}(\mathrm{n})$ is

$$
\begin{aligned}
& f_{p}(x)=\frac{1}{(1-x)\left(1-x^{2}\right)\left(1-x^{3}\right) \cdots\left(1-x^{p}\right)} \\
& =1+\sum_{n=1}^{\infty} P_{p}(n) x^{n}
\end{aligned}
$$

Suppose that $0<\mathrm{x}<1$, so that the product is convergent.

For consider the product

$$
\begin{aligned}
& \left(1+x+x^{2}+\cdots \infty\right)\left(1+x^{2}+x^{4}+\cdots \infty\right) \\
& \left(1+x^{3}+x^{6}+\cdots \infty\right) \cdots\left(1+x^{p}+x^{2 p}+\cdots \infty\right)
\end{aligned}
$$

Any term out of the first, 2nd,--- pth factors may be represented by

$x^{\alpha}, x^{2 \beta}, x^{3 \gamma},---x^{p \theta}$, where $\alpha, \beta, \gamma,----\theta$ are any of the numbers $0,1,2,---, p$.

Thus $\alpha=0, \beta=1, \gamma=2$, the partition $2+\frac{3+3}{2 \text { times }}=2+2.3$ is one partition of 8 and $\alpha=1, \beta=2, \gamma$ $=1$, the partition $1+\frac{2+2}{2 \text { times }}+3=1+2.2+3$ is also one of 8.

If the product of these terms is $x^{n}$, we have $\alpha+2 \beta+3 \gamma$ $+----+p \theta=n$, which is not unique. Hence the coefficient of $x^{n}$ in the product is the number of partitions in which $n$ can be obtained by adding any representation of the numbers 1, 2 ----, p repetitions being allowed. Now the coefficient of $\mathrm{x}^{\mathrm{n}}$ in the product is the number of partitions of $n$ into at most p parts.

But in Remark 3 we have discussed that the number of partitions of $\mathrm{n}$ into $\mathrm{p}$ parts is equal to the number of partitions of $n$ into parts, the largest of which is $p$. So we can say that the number of partitions of $n$ into at most $p$ parts is equal to the number of partitions of $n$ into parts which do not exceed p. i. e. $\mathrm{P}\left(\mathrm{n}, \leq \mathrm{p},{ }^{*}\right)=\mathrm{P}(\mathrm{n}, *, \leq$ p).Hence the Remark.

Here we give the Theorem, which is related to the term $\mathrm{P}(\mathrm{n}, \mathrm{p}, *)$.

Theorem 4: $\mathrm{P}\left(\mathrm{n}, \mathrm{p},{ }^{*}\right)=\mathrm{P}\left(\mathrm{n}-1, \mathrm{p}-1,{ }^{*}\right)+\mathrm{P}(\mathrm{n}-\mathrm{p}, \mathrm{p}$ *).

where asterisk ' $*$ ' means that no restrictions is placed on the nature of the parts.

Proof: We make the p-partitions of $n$ and then we have to distribute n elements into p irrelevant parts, whose sum is $\mathrm{n}$. This can be done by adding a part $\mathrm{p}$ to each partition of n-p into 1 or 2 or 3 or 4 , -------, or p parts. Therefore;

$\mathrm{P}\left(\mathrm{n}, \mathrm{p},{ }^{*}\right)=\mathrm{P}(\mathrm{n}-\mathrm{p}, 1, *)+\mathrm{P}\left(\mathrm{n}-\mathrm{p}, 2,{ }^{*}\right)+\mathrm{P}\left(\mathrm{n}-\mathrm{p}, 3,{ }^{*}\right)+-$ ---+ $\mathrm{P}(\mathrm{n}-\mathrm{p}, \mathrm{p} *)$. Changing $\mathrm{n}$ into $\mathrm{n}-1$ and $\mathrm{p}$ into $\mathrm{p}-1$, we have;

$\mathrm{P}(\mathrm{n}-1, \mathrm{p}-1, *)=\mathrm{P}(\mathrm{n}-\mathrm{p}, 1, *)+\mathrm{P}(\mathrm{n}-\mathrm{p}, 2, *)+\mathrm{P}(\mathrm{n}-\mathrm{p}, 3, *)$ $+----+\mathrm{P}\left(\mathrm{n}-\mathrm{p}, \mathrm{p}-1,{ }^{*}\right)$.

From above we get; $\mathrm{P}\left(\mathrm{n}, \mathrm{p},{ }^{*}\right)=\mathrm{P}\left(\mathrm{n}-1, \mathrm{p}-1,{ }^{*}\right)+\mathrm{P}(\mathrm{n}-\mathrm{p}$, $\left.\mathrm{p},{ }^{*}\right)$. Hence the Theorem.

Example 6: If $\mathrm{n}=11, \mathrm{p}=4$, then $\mathrm{P}(11,4, *)=11$. Again if $n=10, p=3$, then $P(10,3, *)=8$. Finally if $n=7$, $\mathrm{p}=4$, then $\mathrm{P}(7,4, *)=3$.

Therefore we get; $\mathrm{P}(114, *)=11=8+3=\mathrm{P}\left(10,3,{ }^{*}\right)$ $+\mathrm{P}(7,4, *)$. 


\section{Conclusion}

In this study, we have found the number of partitions of $\mathrm{n}$ when $\mathrm{n}=1$ to 17 by using Macmahon's table. We have verified the remark 1 when $\mathrm{n}=8$, and have found that the function $1+\sum_{m=1}^{\infty} \frac{x^{m^{2}}}{\left(1-x^{2}\right)\left(1-x^{4}\right) \ldots\left(1-x^{2 m}\right)}$ enumerates all self-conjugate partitions of $\mathrm{n}$, and have also proved the Euler's Theorems with the second parameter $\mathrm{z}=1$ and $\mathrm{x}$ respectively. We have established the Theorem 3 with the help of Euler's generating function for P(n) and have verified the Theorem 4 with numerical example.

\section{Acknowledgment}

It is a great pleasure to express my sincerest gratitude to my respected teacher Professor Md. Fazlee Hossain, Department of Mathematics, University of Chittagong,
Bangladesh. I will remain ever grateful to my respected teacher Late Professor Dr. Jamal Nazrul Islam, JNIRCMPS, University of Chittagong, Bangladesh.

\section{References}

[1] Andrews, GE, An Introduction to Ramanujan's Lost Notebook, Amer. Math. Monthly, 86, 1979. 89-108.

[2] Burn, R.P. (1964). A Pathway into Number Theory, $2^{\text {nd }}$ Edition.

[3] Hardy G.H and Wright, E.M. Introduction to the Theory of Numbers, $4^{\text {th }}$ Edition, Oxford, Clarendon Press, 1965.

[4] IVAN and NIVEN, An introduction to the theory of Numbers, Fifth edition (1969).

[5] Macmahon, Combinatory analysis, Ann Arbor, Michigan, University of Michigan Library, 2005.

[6] S.BARNARD and J.M. CHILD, Higher Algebra, Macmillan (1967).

[7] S.Ramanujan (1919), Some properties of P(n),number of partitions of n, Proc. of the Cam. Philo. Society X1X: 207-210.

[8] Sabuj Das and Haradhan Kumer Mohajan, Generating Functions For X(n) an Y(n), American Review of Mathematics and Statistics, Vol. 2 No. 1, March 2014. 